\title{
Digital radiology and Picture Archiving and Communication System (PACS)
}

Digital imaging systems for plain radiography

Springer; 2013. p. 137-158. DOI: 10.1007/978-1-4614-5067-2_9

Luís Lança and Augusto Silva

\section{Abstract}

Plain radiography still accounts for the vast majority of imaging studies that are performed at multiple clinical instances. Digital detectors are now prominent in many imaging facilities and they are the main driving force towards filmless environments. There has been a working paradigm shift due to the functional separation of acquisition, visualization, and storage with deep impact in the imaging workflows. Moreover with direct digital detectors images are made available almost immediately. Digital radiology is now completely integrated in Picture Archiving and Communication System (PACS) environments governed by the Digital Imaging and Communications in Medicine (DICOM) standard. In this chapter a brief overview of PACS architectures and components is presented together with a necessarily brief account of the DICOM standard. Special focus is given to the DICOM digital radiology objects and how specific attributes may now be used to improve and increase the metadata repository associated with image data. Regular scrutiny of the metadata repository may serve as a valuable tool for improved, cost-effective, and multidimensional quality control procedures.

Keywords: Digital radiology; Picture archiving; Communication; PACS; Digital detectors; Filmless environment; Direct digital detector images; DICOM; Metadata repository; Image data; Cost-effectiveness; Quality control. 